\title{
MONTGOMERY-SAMELSON SINGULAR FIBERINGS OF SPHERES ${ }^{1}$
}

\section{P. L. ANTONELLI}

1. Introduction. The theory of Montgomery-Samelson singular fiberings is concerned with maps which are bundle maps except on a singular set, over which the fibers have been pinched to points. The original definition is in Montgomery-Samelson [8], and related ones are in Conner-Dyer [6], Mahowald [7], Antonelli [1], and ChurchTimourian [5]. ${ }^{2}$ We will define a map $f$ to be an MS-fibering of manifolds if

(1) $f:\left(M^{n}, A\right) \rightarrow\left(N^{p}, f(A)\right)$ is open and onto;

(2) $f^{-1}(f(A))=A, A$ is closed and nonempty, and $f / A$ is a homeomorphism;

(3) $f: M^{n}-A \rightarrow N^{p}-f(A)$ is a locally trivial fibering with fiber $F^{n-p}$ and $M^{n}-A$ is connected.

We also require $M^{n}, N^{p}, A, f(A)$ and $F^{n-p}$ to be closed connected orientable and triangulable manifolds with $n>p$. The fibering will be smooth if $f$ and these manifolds are $C^{\infty}$-differentiable and if the inclusions $A \rightarrow M$ and $f(A) \rightarrow N$ are $C^{\infty}$-imbeddings.

Investigations in the theory of transformation groups led Montgomery and Samelson to conjecture in 1946 that if $M^{n}$ is a sphere and $f: M^{n} \rightarrow N^{p}$ is a differentiable MS-fibering (where $N^{p}$ has possibly nonempty boundary and $A$ is not assumed to be a manifold), then the singular set $A$ is a homology sphere. Mahowald [7] showed essentially that, if $n \leqq 4$ and $M^{n}$ is a $Z_{2}$-cohomology sphere, then $A$ is also a $Z_{2}$-cohomology sphere. Prior to this Conner-Dyer [6] obtained similar results for arbitrary $n$, but with the additional requirement that the fiber be a $Z_{2}$-cohomology sphere. Differentiability was not assumed in either paper and the singular fiberings involved spaces more general than those we consider here.

The purpose of this note is to indicate recent progress on a form of the MS-conjecture nearer to the original.

\section{Main results.}

THEOREM 1. Let $f:\left(S^{n}, A\right) \rightarrow\left(N^{p}, f(A)\right)$ be an MS-fibering of manifolds; let $A$ and $f(A)$ be locally flatly imbedded in $S^{n}$ and $N^{p}$, and let

Received by the editors March 22, 1968 and, in revised form, October 16, 1968.

1 This work was partially supported by NSF contract GP-5420.

${ }^{2}$ The author would like to thank P. T. Church and J. G. Timourian for access to copies of their papers on singular fiberings before publication. 
$\pi_{1}\left(N^{p}-f(A)\right)$ act simply on $H_{*}(F ; Z)$. Then $A$ is an integral homology $(2 p-n-2)$-sphere.

As a corollary to Theorem 1 we have

Theorem 2. If $f:\left(S^{n}, A\right) \rightarrow\left(N^{p}, f(A)\right)$ is a smooth $M S$-fibering of manifolds, then $A$ is an integral homology (2p-n-2)-sphere.

Theorem 2, therefore, affirms the MS-conjecture in the case where $A$ and $f(A)$ are smooth manifolds and $N^{p}$ is without boundary. Using (2) we can also prove (assuming the Poincaré hypothesis if $\operatorname{dim} A$ is 3 or 4 ).

Theorem 3. If $f:\left(S^{n}, A\right) \rightarrow\left(S^{p}, f(A)\right)$ is a smooth $M S$-fibering of manifolds with $A$ a simply connected $q$-manifold, then $f$ is topologically equivalent to the $(q+1)$-fold suspension of a locally trivial fiber map $S^{2 m-1} \rightarrow S^{m}, m=2,4,8$.

Results similar to (3) may be found in Antonelli [1]. Global results in the case where the singular set consists of isolated points may be found in Antonelli [2], [3].

3. Outline of Proofs of Theorems 2 and 3. We first prove Theorem 3 using Theorem 2. It is clear that $A, f(A)$ are homotopy spheres. Assuming the Poincaré conjecture true in $\operatorname{dim} 3,4$ we obtain sphere pairs $\left(S^{n}, A\right)$ and $\left(S^{p}, f(A)\right)$. Since $A, f(A)$ are locally flatly imbedded we may conclude that they are standard sphere pairs, provided codim $(A)$ and $\operatorname{codim}(f(A)) \geqq 3$, via a theorem of Stallings [9].

Suppose for the moment that this is the case. Since we have sphere pairs there is a pair of antipodal points $\{p, q\}$ in $A$ which is carried onto a pair of antipodal points $\left\{p^{1}, q^{1}\right\}$ in $f(A)$ by the homeomorphism $f / A$. Removing these pairs of points and carefully deforming the restriction map $S^{n}-\{p, q\} \rightarrow S^{p}-\left\{p^{1}, q^{1}\right\}$ we obtain an MSfibering of $S^{n-1}$ onto $S^{p-1}$ with singular set a standard $(q-1)$-sphere. The suspension of this new fibering is topologically equivalent to $f$. Proceeding in this way we see that $f$ is topologically equivalent to the $(q+1)$-fold suspension of a locally trivial fibre map $S^{2 m-1} \rightarrow S^{m}, m=2$, 4,8 . See [1] for more details.

In order to prove codim of $A$ and $f(A) \geqq 3$, it will suffice to show codim $f(A) \geqq 3$. If $\operatorname{codim} f(A)=1$, then $f(A)$ separates $S^{p}$ and implies that $A$ separates $S^{n}$. But this is impossible since codim $A \geqq$ codim $f(A)+1$ (because $\operatorname{dim} F^{n-p} \geqq 1$ ).

Suppose codim $f(A)=2$. Removing singular sets and deforming, we obtain a fibering $L \rightarrow K$ of compact sets with projection map $f / L$, $L=f^{-1}(K)$. This map is open so by a theorem of Whyburn [12] it 
induces an epimorphism on 1-dimensional rational homology if $H_{1}(L)$ is finite-dimensional as a vector space. From Alexander duality applied to the pairs $\left(S^{p}, f(A)\right)$ and $\left(S^{n}, A\right)$ we obtain $\operatorname{dim} H_{1}(K)=1$ and $\operatorname{dim} H_{1}(L)=0$ - a contradiction. Therefore, $\operatorname{codim} f(A) \geqq 3$, as desired.

REMARK. Using Theorem 3 and what is essentially the standard argument for the suspensions of Hopf maps, it follows that $f$ is an essential map. However, it is known that it is not necessary for fiber maps $S^{2 m-1} \rightarrow S^{m}$ to be topologically equivalent to the Hopf maps except in the case $m=2$.

Proof of (2). Since codim $A \geqq 3$ it follows from a well-known transversality argument that $S^{n}-A$ is simply connected. Applying the fiber-homotopy sequence we see that $N^{p}-f(A)$ is also simply connected. Therefore, $\pi_{1}\left(N^{p}-f(A)\right)$ acts simply on $H_{*}(F ; Z)$ and the desired conclusion follows from (1).

4. Proof of Theorem 1. By local flatness there exist small open sets $V$ around $x$ in $A$ with $(V, V \cap A)$ homeomorphic to $\left(R^{n}, R^{q}\right)$. Therefore the inclusion of a small linking $S^{n-q-1}$ in $V-A$ is a homotopy equivalence; the analogous result holds for suitable open $W$ around $f(x)$ in $f(A)$, and a small linking sphere $S^{p-q-1}$. Furthermore, putting $L^{n-q-1}=f^{-1}\left(S^{p-q-1}\right)$, it follows from consideration of the homotopy sequences of the fibering (over $S^{p-q-1}$ and $W-f(A)$ ) that the inclusion $L \subset f^{-1}(W)-A$ is a homotopy equivalence. Since each $V$ contains an $f^{-1}(W)$ and conversely, it follows easily that $L$ is a homotopy $(n-q-1)$-sphere. From [4], $F^{n-p}$ is a homotopy 1,3 or 7 sphere. $^{3}$

The simple action hypothesis now allows one to make use of the exactness of the Gysin sequence

$$
\cdots \rightarrow H_{c}^{i}\left(N^{p}-f(A)\right) \rightarrow H_{c}^{i+r+1}\left(N^{p}-f(A)\right) \stackrel{f^{*}}{\rightarrow} H_{c}^{i+r+1}\left(S^{n}-A\right) \rightarrow \cdots
$$

with $r=n-p$ and coefficients in any field $Z_{p}$. The subscript $c$ denotes compact supports. An argument similar to Conner-Dyer, [6, Theorem 2.1] now implies that $A$ is a $Z_{p}$-cohomology $(n-k(n-p+1))$-sphere for some positive integer $k$. Indeed, the fact that $n-p=1,3$ or 7 implies that $k=2$ and so $A$ is $2 p-n-2$ dimensional. The universalcoefficient theorem and the fundamental theorem of abelian groups now imply that $A$ is an integral homology $(2 p-n-2)$-sphere as desired.

${ }^{3}$ Our original argument was due to J. G. Timourian. Its present form was suggested by the referee. 
REMARK. Theorem 1 can be proved using the layering phenomenon of Church-Timourian [5, Theorem 1.3]. In fact, $A$ can be shown to be a locally orientable $Z_{2}$-generalized $(2 p-n-2)$-manifold. Further results on local structure theory of singular fiberings may be found in Timourian [10], [11] and Church-Timourian [5].

The author is grateful for the referee's remarks, in particular those concerning the first part of the proof of Theorem 1 .

\section{BiBLIOGRAPHY}

1. P. L. Antonelli, Structure theory for Montgomery-Samelson fiberings between manifolds. I, Canad. J. Math. 21 (1969), 170-179.

2. - - Structure theory for Montgomery-Samelson fiberings between manifolds. II, Canad. J. Math. 21 (1969), 180-186.

3. - Smooth MS-fiberings with finite singular sets, Canad. J. Math. (to appear).

4. W. Browder, Higher torsion in H-spaces, Trans. Amer. Math. Soc. 108 (1963), $353-375$.

5. P. T. Church and J. G. Timourian, Fiber bundles with singularities, J. Math. Mech. 18 (1968), 71-90.

6. P. E. Conner and E. Dyer, On singular fiberings by spheres, Michigan Math. J. 6 (1959), 303-311.

7. M. Mahowald, Singular fiberings, Canad. J. Math. 15 (1963), 214-219.

8. D. Montgomery and H. Samelson, Fiberings with singularities, Duke Math. J. 13 (1946), 51-56.

9. J. Stallings, On topologically unknotted spheres, Ann. of Math. (2) 77 (1963), 490-503.

10. J. G. Timourian, Fiber bundles with discrete singular set, J. Math. Mech. 18 (1968), 61-70.

11. - Maps with discrete branch sets between manifolds of codimension one, Canad. J. Math. (to appear).

12. G. Whyburn, The mapping of Betti groups under interior transformations, Duke Math. J. 4 (1938), 1-8.

The University of Tennessee 